\title{
Atomistic Study of the Effect of Magnesium Dopants on the Strength of Nanocrystalline Aluminum
}

\author{
Amirreza Kazemi, Shengfeng Yang* \\ Department of Mechanical and Energy Engineering, Indiana University Purdue University Indianapolis, \\ Indianapolis, IN, 46202, USA
}

\begin{abstract}
Atomistic simulations have been used to study the deformation mechanisms of nanocrystalline pure $\mathrm{Al}$ and $\mathrm{Al}-\mathrm{Mg}$ binary alloys. Voronoi tessellation was used to fully create a three dimensional polycrystalline model with a grain size of $10 \mathrm{~nm}$, while hybrid Monte Carlo and molecular dynamic simulations were used to achieve both mechanical and chemical equilibrium in nanocrystalline Al-5 at. \% Mg. The results of tensile tests show an improved strength, including the yield strength and ultimate strength, through doping 5 at.\% $\mathrm{Mg}$ into nanocrystalline aluminum. The results of atomic structures clearly reveal the multiple strengthening mechanisms related to doping in Al-Mg alloys. At the early deformation stage, up to an applied strain of 0.2 , the strengthening mechanism of dopants exhibits as dopant pinning grain boundary (GB) migration. However, at the late deformation stage, which is close to failure of nanocrystalline materials, dopants can prohibit the initiation of intergranular cracks and also impede propagation of existing cracks along the GBs, thus improving the flow stress of Al-Mg alloy.
\end{abstract}

Keywords: Aluminum-Magnesium alloy; Atomistic simulations; Grain boundary segregation; Strengthening mechanisms; Dopants pinning grain boundary migration

\section{Introduction}

Nanocrystalline (NC) materials have been widely used in various applications due to their extraordinary material properties such as high ultimate strength and superior hardness $[1,2]$. Due to the large volume fraction of high-energy interfacial regions in NC materials, their low thermal stability [3] and low ductility [4] has greatly limited their applications. Alloying NC materials with different elements has been an effective strategy to improve thermal stability at elevated temperatures, enhance ductility, and further increase strength. The alloying atoms are either randomly distributed in the grain interior as solid solutions or segregated to grain boundaries (GB). Dopants segregated to grain boundaries (GBs) change the chemistry at GBs, which could lead to transformations of GB complexions [5-9] and thus impact the material properties [10-15].

For Al-Mg alloys, researchers have identified that $\mathrm{Mg}$ dopants can improve the mechanical properties of materials, including yield strength and strain hardening, through the solid solution strengthening $[16,17]$ and precipitates pinning GBs $[18,19]$. In addition to those mechanisms, Pun et al. [2] found that GB segregation strengthening also contributes to the improved strength and hardness of Al$\mathrm{Mg}$ alloys. Being that $\mathrm{Mg}$ atoms have a strong tendency to segregate to GBs, GB segregation strengthening has a larger contribution than solid solution strengthening in Al-Mg alloys. However, there are currently no atomic level mechanisms available for clearly explaining how the effect of $\mathrm{Mg}$ dopants segregated to GBs leads to improvement of the strength of NC Al. Moreover, it is well known that doping $\mathrm{Mg}$ can stabilize the grain growth of $\mathrm{NC} \mathrm{Al}$ against elevated temperature [18]. However, it is unclear how $\mathrm{Mg}$ dopants segregated to GBs can affect the stability of the grain sizes of $\mathrm{NC}$ Al during the deformation process.

In this study, we used atomistic simulations to explore the underlying atomic-level mechanisms of $\mathrm{GB}$ segregation strengthening of $\mathrm{NC} \mathrm{Al}$ by doping $\mathrm{Mg}$ atoms. Fully three dimensional (3D) polycrystalline models for both pure $\mathrm{Al}$ and $\mathrm{Al}-5 \% \mathrm{Mg}$ were used to avoid the geometric limitations of 2D or 3D columnar polycrystalline models [20]. A hybrid MC/MD method was used to obtain the

*Corresponding author. E-mail address: yangsf@iupui.edu (S. Yang). 
equilibrium distribution of $\mathrm{Mg}$ atoms in the $\mathrm{Al}-\mathrm{Mg}$ alloy model. Tensile simulations were conducted on both $\mathrm{Al}$ and $\mathrm{Al}-\mathrm{Mg}$ alloys to investigate the effect of $\mathrm{Mg}$ dopants on the mechanical properties of $\mathrm{NC} \mathrm{Al}$ and the underlying mechanisms. The atomic-level mechanisms for failure of $\mathrm{NC} \mathrm{Al}$ and $\mathrm{Al}-\mathrm{Mg}$ alloy under tensile tests were also investigated through atomistic simulations.

\section{Computational details}

The NC Al models were created using the Voronoi tessellation procedure. The dimensions of the simulation cube were set to $200 \AA, 200 \AA$, and $200 \AA$ along the x, y, and z directions, respectively. Twelve grain centers were placed randomly within the simulation box. To effectively model the average behavior of bulk polycrystalline samples, the crystal orientation for each grain was randomly assigned. Al atoms were used to fill each grain according to its crystal orientation. The 3D polycrystal model contained 483,197 atoms and the average grain size was estimated to be about $10 \mathrm{~nm}$ using a spherical grain assumption. Periodic boundary conditions were applied in all directions. In order to avoid unphysical interaction between neighboring atoms, atoms that are closer than $0.2 \AA$ from another atom were removed from the model.

A Finnis-Sinclair (F-S) type of interatomic potential [21] was used to describe the Al-Al, Mg-Mg, and Al-Mg interactions due to the better reproducibility of thermal and mechanical properties of Al-Mg alloys. The bulk phase diagram of Al-Mg was better reproduced by this potential than other available Al$\mathrm{Mg}$ potentials $[22,23]$. Specifically, the solidus and liquidus lines are correctly reproduced by this F-S potential at the Al-rich side of the phase diagram [21] and the composition of Al-5at.\%Mg for this work is within in this range.

To create NC Al-Mg models with an equilibrium distribution of $\mathrm{Mg}$ atoms, we employed hybrid Monte Carlo/molecular dynamics (MC/MD) simulations to introduce $\mathrm{Mg}$ atoms into the $\mathrm{NC} \mathrm{Al}$ model, rather than directly placing the $\mathrm{Mg}$ atoms at the GBs or the grain interior [24]. Hybrid MC/MD [25, 26] has been a useful tool to help to achieve an equilibrium distribution of dopants in matrix atoms. In hybrid MC/MD simulations, the atomic structural relaxations are realized by MD running, and the MC scheme samples the semi-grand canonical ensemble. $\mathrm{MC}$ swaps of $\mathrm{Al}$ atoms with $\mathrm{Mg}$ atoms are performed, and the swap probability is dictated by the Metropolis criterion in specified temperatures. All atoms move according to the regular MD time integration and the time step is $0.1 \mathrm{fs}$. The hybrid MC/MD simulation is considered to reach an equilibrium if the fluctuation of total energy over the last 10,000 steps is less than $0.5 \%$. A chemical potential difference of $1.9 \mathrm{eV}$ between $\mathrm{Mg}$ and $\mathrm{Al}$ was used in the hybrid MC/MD simulation to achieve 5 at.\% $\mathrm{Mg}$ in our model. The 5 at.\% of $\mathrm{Mg}$ dopants was chosen in this study because Al-Mg alloys with a $\mathrm{Mg}$ content in the range of 1-5 at.\% have been widely used in practical engineering applications. The model was relaxed to achieve both mechanical and chemical equilibrium, while keeping the system temperature at $300 \mathrm{~K}$ and the pressure equal to zero. During the uni-axial tensile simulation, straining was carried out along the x-direction, while the other two directions were free to expand or shrink. A constant strain rate was applied to both $\mathrm{NC} \mathrm{Al}$ and Al-Mg alloys. A small time step of $0.1 \mathrm{fs}$ was used for time integration in solving the equation of motion during the simulations. The tensile simulations were carried out using NPT ensemble with the temperature of $300 \mathrm{~K}$ and zero pressure. All simulations were performed using the LAMMPS [27]. The software of OVITO [28] was used for the visualization of the atomic structures.

\section{Results}

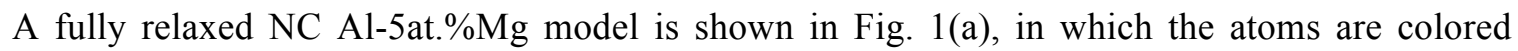
according to their common neighbor analysis (CNA) values. The Al atoms with fcc structure are colored in green; the $\mathrm{Al}$ atoms at GBs are colored in blue. Specifically, the $\mathrm{Mg}$ atoms are colored red to clearly show their distribution. In order to visualize the distribution of $\mathrm{Mg}$ atoms inside the model, a cross- 
section of the 3D model is shown in Fig. 1 (b), which clearly shows that the density of Mg atoms is larger at GBs than in the grains. Through analyzing different slices of the model, we found that the strong segregation of $\mathrm{Mg}$ at $\mathrm{Al} \mathrm{GB}$ exists in the entire 3D model. This observation is in agreement with the research of Pun et al. [2], who also observed that $\mathrm{Mg}$ tends to segregate at Al GBs and the segregation amount increases with the increasing annealing temperature and time.
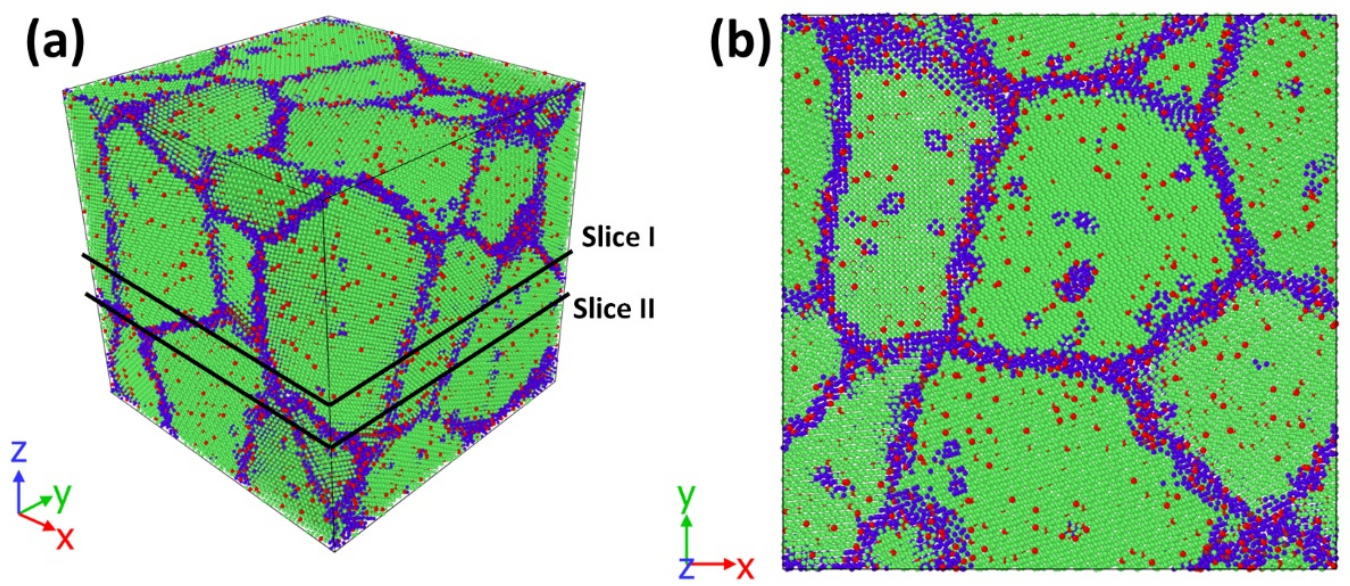

Fig. 1 A fully relaxed nanocrystalline Al-5at.\%Mg alloy (a) 3D model and (b) Slice II: a cross-section parallel to $\mathrm{x}-\mathrm{y}$ plane. The $\mathrm{Al}$ atoms with fcc structure are colored green; the $\mathrm{Al}$ atoms at $\mathrm{GBs}$ are colored blue; and the red atoms with a larger size are $\mathrm{Mg}$ atoms.

The tensile tests were conducted on both the NC pure Al and Al-Mg alloy models at $300 \mathrm{~K}$. In order to consider the effect of the applied strain rate, two different constant strain rates, i.e., $10^{9} \mathrm{~s}^{-1}$ and $10^{10} \mathrm{~s}^{-1}$, were applied. The obtained stress-strain curves are plotted for pure Al using black solid and dot lines, and Al-Mg alloys are plotted using red dash and dash-dot lines, as shown in Fig. 2. The solid and dash curves are obtained at a strain rate of $10^{9} \mathrm{~s}^{-1}$, while the dot and dash-dot lines are from the strain rate of $10^{10} \mathrm{~s}^{-1}$. The results clearly show that the Al-5 at.\% Mg alloy has a larger strength, including yield strength and ultimate strength, and better ductility compared to the pure Al. Similar findings can be obtained from the simulation results under these two different applied strain rates. A similar strengthening effect of $\mathrm{Mg}$ atoms has been reported in the literature [2, 16]. Specifically, Pun et al. [2] found that both the strength and hardness of NC Al can be improved by doping Mg atoms. Lee et al. [16] reported a simultaneous improvement in both the strength and the ductility for Al-Mg alloy as compared to pure Al. 


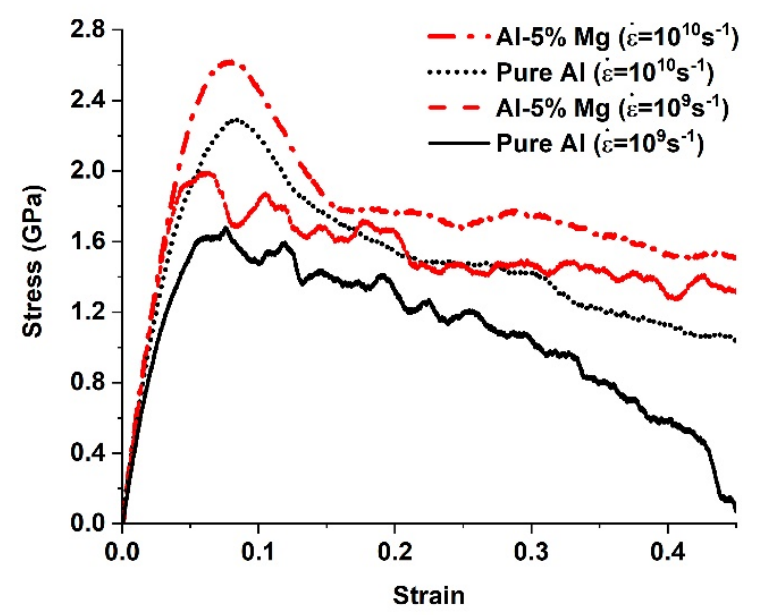

Fig. 2 Stress-strain curves obtained from tensile simulations of nanocrystalline pure $\mathrm{Al}$ and $\mathrm{Al}-5 \% \mathrm{Mg}$ alloy at two different strain rates

Atomic structures were analyzed to understand the strengthening effect of $\mathrm{Mg}$ dopants. Two slices were cut from both pure $\mathrm{Al}$ and $\mathrm{Al}-\mathrm{Mg}$ alloy $3 \mathrm{D}$ models subject to a constant strain rate of $10^{9} \mathrm{~s}^{-1}$. The origin of the coordinate system, i.e., point $(0,0,0)$, is located in the center of the model. Slice I was cut parallel to the xy-plane at the position of $z=-4 \AA$, as shown in Fig. 1(a). The slice from the pure Al model was cut at the same location as that for the Al-Mg alloy model. The thickness for these two slices are 6 Å. Fig. 3 shows structures of Slice I for pure Al and Al-Mg alloys at different applied strains up to 0.2. The top row of subfigures show the atomic structures of Slice I at four different applied strains for the $\mathrm{NC}$ pure Al, while the bottom row are for Al-Mg alloys. The Al atoms are colored according to their $\mathrm{CNA}$ values. The $\mathrm{Al}$ atoms with fcc, hcp, and disordered structure are colored in green, brown, and blue, respectively. The red atoms with a larger size are $\mathrm{Mg}$ atoms. The strips of brown atoms in Fig. 3(c), (d), (f), (g), and (h) are stacking faults with the hcp structure left by dislocation partials. The blue disordered regions are GB regions or amorphous regions. Fig. 4 shows the atomic structure of Slice II that was taken at a different position $(\mathrm{z}=-30 \AA)$ but was still parallel to the xy-plane, as shown in Fig. 1(a). The applied strain is up to 0.4 in Fig. 4 . The thickness for this slice is $10 \AA$. The same color scheme for different atoms was used in this figure. The numbers from 1 to 3 in both Fig. 3 and Fig. 4 were used to label three different grains in the models in order to track the motions of these three grains during the tensile simulations. 

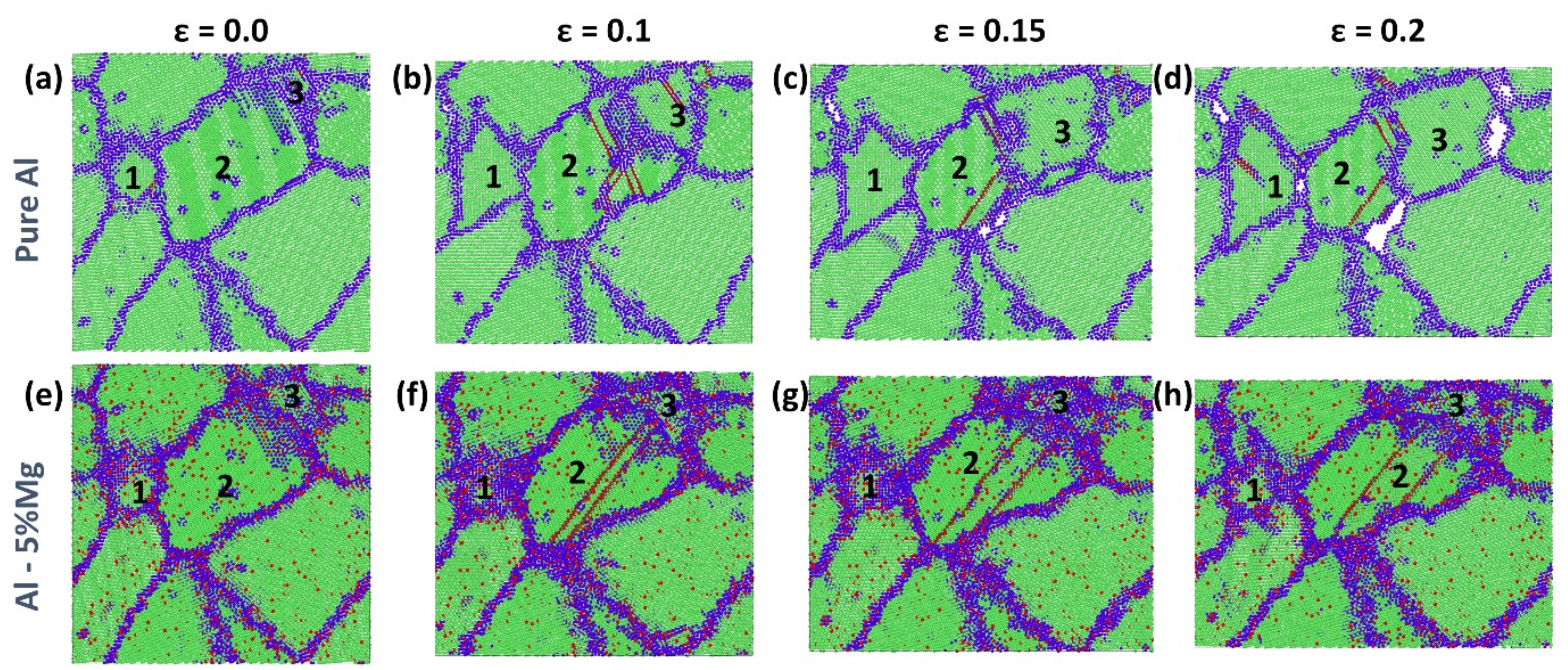

Fig. 3 Atomic structure for Slice I of the nanocrystalline pure $\mathrm{Al}$ and $\mathrm{Al}-5 \mathrm{at} . \% \mathrm{Mg}$ alloy models at different applied strains. In both cases, the same slice parallel to the xy-plane is shown. Pure $\mathrm{Al}(\mathrm{a})-(\mathrm{d})$ and Al-Mg alloy (e)-(h) are plotted at the applied strains of $0.0,0.1,0.15$, and 0.2 respectively.

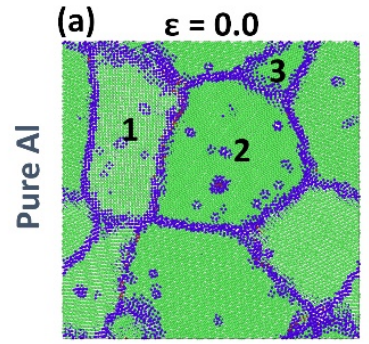

(e)

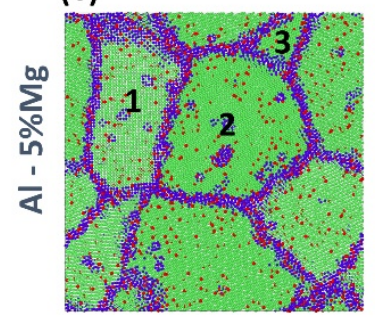

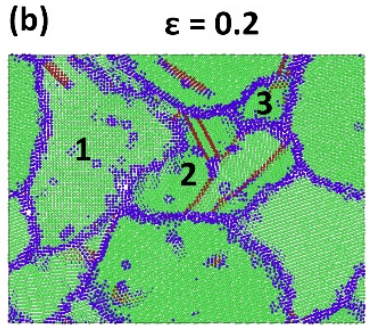

(f)

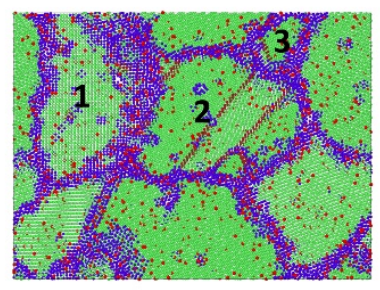

(c) $\varepsilon=0.3$

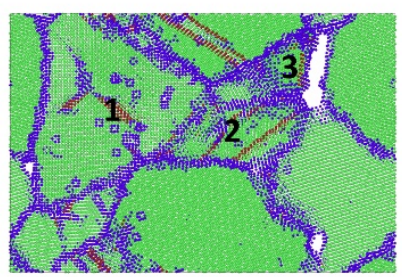

(g)

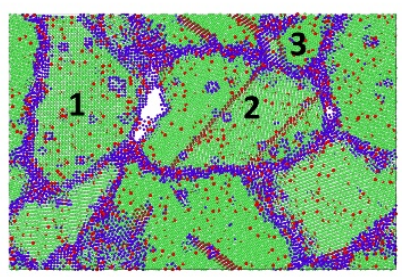

(d) $\quad \varepsilon=0.4$

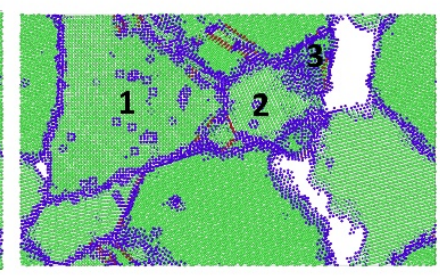

(h)

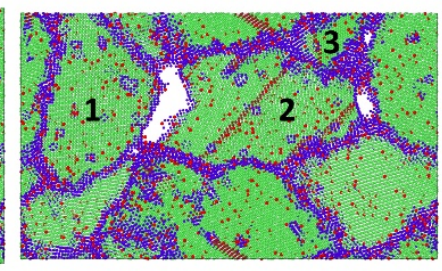

Fig. 4 Atomic structure for Slice II of the nanocrystalline pure $\mathrm{Al}$ and $\mathrm{Al}-5 \mathrm{at} . \% \mathrm{Mg}$ alloy models at different applied strains. Pure Al (a) - (d) and Al-Mg alloy (e)-(h) are plotted at the applied strains of 0.0, $0.2,0.3$, and 0.4 respectively. The number from 1 to 3 are used to label and track these three grains during the tensile loading. 
(a)

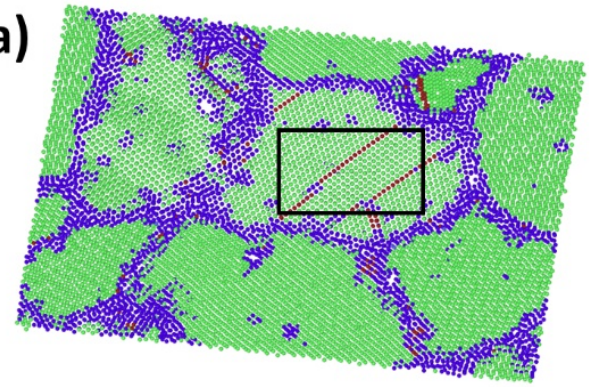

(b)

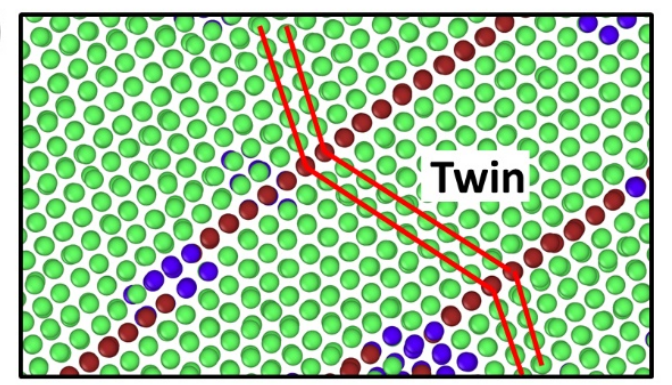

Fig. 5 Atomic structure of the observed deformation twinning in Al-5at.\%Mg alloy at the applied strain of 0.2: (a) Slice II in Fig. 4(f) which are rotated to clearly show the twinning, (b) a close-up view of the deformation twin.

\section{Discussion}

In order to identify the mechanisms for improving the strength of $\mathrm{NC} \mathrm{Al}$ through doping $\mathrm{Mg}$ atoms, we monitored the motions and size changes of grains during the tensile simulations. Different GB induced deformation mechanisms were identified, including dislocation nucleation from GB and deformation twinning in both pure $\mathrm{Al}$ and $\mathrm{Al}-\mathrm{Mg}$ alloy. For example, Fig.5 shows the atomic structure of the deformation twin observed in Al-5at\% $\mathrm{Mg}$ alloy at the applied strain of 0.2 . The results of pure $\mathrm{Al}$ show that the GB migration was frequently observed in pure $\mathrm{Al}$ in order to accommodate the deformation during the tensile loading. However, it was difficult to find GB migrations in the Al-Mg alloys. As shown in Fig. 3(a)-(d) for pure Al, the sizes of Grain \#1 and \#3 increased with the increasing strain, while the size of Grain \#2 decreased. The comparison of the sizes of these three grains clearly shows that the size ratio between grains changed during the tensile loading due to the migrations of GBs between the neighboring grains. However, for the same slice in the Al-Mg alloy model shown in Fig. 3(e) to (h), the change in the size ratio between Grains \#1, \#2, and \#3 is very small and negligible.

Similar findings can be obtained from analyzing the results of a different slice shown in Fig. 4. The size ratio between Grain \#1 and Grain \#2 changed for pure Al by Fig. 4 (a)-(d) while the change in the size ratio is much smaller for the Al-Mg alloy as shown in Fig. 4 (e)-(h). This dopant pinning effect on GB migration agrees well with a study by Rahman et al. [29]. A simple tilt GB model was used in their study and artificial driving force (ADF) techniques were used to induce GB motions. They found that a strong dopant pinning effect was observed at different misorientations. In this study, we used fully 3D nanocrystalline models to reveal the Mg dopant pinning effects on GB migration in NC Al. All these results clearly indicate that the addition of $\mathrm{Mg}$ atoms can effectively impede the GB migration in $\mathrm{NC} \mathrm{Al}$, and hence improve its strength at the early deformation stage up to a strain of 0.2 .

The analysis of the failure process of pure $\mathrm{Al}$ and $\mathrm{Al}-\mathrm{Mg}$ indicates that $\mathrm{Mg}$ atoms can also effectively prohibit the nucleation of intergranular cracks in NC Al. The ultimate failure of both NC pure $\mathrm{Al}$ and $\mathrm{Al}-\mathrm{Mg}$ alloy were caused by the initiation of intergranular cracks from the regions close to triple junctions and their propagation along the GBs in the materials. This failure mechanism agrees well with the finding from a study by Hocker et al. [30]. They observed inter-granular fracture in both pure Al and Al-Mg alloy but didn't find any difference in tensile strength between them. However, in our study, we found that the strength of the Al-Mg alloy is higher than pure Al. The strengthening mechanism includes the effect of dopants on prohibiting crack initiation in Al-Mg alloys. As shown in Fig. 3 (c) for pure Al at the applied strain of 0.15 , cracks nucleated at triple junctions near Grain \#1 and \#2 in pure Al. However, no cracks were observed for Al-Mg alloys at the strain of 0.15 and even up to 0.2 as shown in Fig. 3 (g) 
and (h). The addition of $\mathrm{Mg}$ dopants leads to the formation of larger disordered GB regions and triple junction regions compared to the pure Al model, as shown in the comparison between Fig. 3(b) and 3(f). The larger disordered triple junction regions can better accommodate the deformation at triple junctions and hence prohibit the initiation of cracks from triple junctions, as shown in Fig. 3 (c) and Fig. 3 (g).

Moreover, Mg atoms segregated to GBs can affect the flow stress of NC Al through impeding the propagation of existing intergranular cracks. As shown in Fig. 4 (c) and (d), the crack near Grain \#3 is propagating quickly in pure $\mathrm{Al}$ when the applied strain increases from 0.3 to 0.4 . Compared to pure $\mathrm{Al}$, the propagation of the crack between Grain \#1 and \#2 in Al-Mg alloys is much slower with the strain increased from 0.3 to 0.4 , as shown in Fig. $4(\mathrm{~g})$ and (h). The larger amorphous GB regions induced by segregation of $\mathrm{Mg}$ atoms act as stronger barriers to the propagating cracks in the Al-Mg alloy. All these results suggest that $\mathrm{Mg}$ dopants segregated to GBs can strengthen the $\mathrm{NC}$ Al through effectively prohibiting the nucleation of intergranular cracks and impeding the propagation of existing cracks.

The stability of nanocrystalline materials can be improved by GB doping to suppress the driving force for grain coarsening. For example, the experiments of sputter-deposited Al alloy [18] show that the grain size of Al-Mg alloys was found to be much smaller than that of pure Al, though they were treated at the same conditions. Doping with different elements has been widely used to stabilize the grain size of nanocrystalline materials against the increase of temperature. In this study, we illustrate that $\mathrm{Mg}$ dopants can effectively stabilize the grain size during the deformation process of $\mathrm{NC} \mathrm{Al} \mathrm{due} \mathrm{to} \mathrm{the} \mathrm{pinning} \mathrm{effect}$ of dopants on GB migrations. This result indicates that GB doping could be an effective strategy for controlling the grain size of nanocrystalline materials during manufacturing processes that utilize deformations, such as severe plastic deformation (SPD) techniques.

\section{Conclusions}

In conclusion, we found that the strength of $\mathrm{NC} \mathrm{Al}$ was improved by doping 5at.\% $\mathrm{Mg}$, through use of atomistic simulations. GB migration can accommodate the deformation during the tensile simulations of nanocrystalline Al. The addition of $\mathrm{Mg}$ dopants can help to effectively impede the GB migration in nanocrystalline Al, and thus improve its strength at the early deformation stage. Moreover, our results clearly show that the dopants segregated to GBs can effectively prohibit the nucleation of intergranular cracks and hinder the propagation of existing intergranular cracks along GBs. This effect contributes to the improvement of -flow stress for Al-Mg alloys, as compared to pure Al. Our results also show the promising application of doping in controlling the grain size, and hence improving the stability of nanocrystalline materials during deformation processes.

\section{Acknowledgements}

This work is financially supported by the faculty start-up funding provided by Purdue School of Engineering and Technology at Indiana University Purdue University Indianapolis. This research was supported in part by Lilly Endowment, Inc., through its support for the Indiana University Pervasive Technology Institute, and in part by the Indiana METACyt Initiative. The Indiana METACyt Initiative at IU was also supported in part by Lilly Endowment, Inc. This work used the Extreme Science and Engineering Discovery Environment (XSEDE) under award allocation number TG-MSS180015.

\section{Reference}

1. I.A. Ovid'ko, R.Z. Valiev, and Y.T. Zhu, Prog. Mater. Sci., 94, 462 (2018). 
2. S.C. Pun, W.B. Wang, A. Khalajhedayati, J.D. Schuler, J.R. Trelewicz, and T.J. Rupert, Mater. Sci. Eng. A, 696, 400 (2017).

3. T. Chookajorn, H.A. Murdoch, and C.A. Schuh, Science, 337, 951 (2012).

4. M. Rajagopalan, M.A. Bhatia, M.A. Tschopp, D.J. Srolovitz, and K.N. Solanki, Acta Mater., 73, 312 (2014).

5. P.R. Cantwell, M. Tang, S.J. Dillon, J. Luo, G.S. Rohrer, and M.P. Harmer, Acta Mater., 62, 1 (2014).

6. T. Frolov, and Y. Mishin, J. Chem. Phys., 143, 044706 (2015).

7. W.D. Kaplan, D. Chatain, P. Wynblatt, and W.C. Carter, J. Mater. Sci., 48, 5681 (2013).

8. T. Hu, S.F. Yang, N.X. Zhou, Y.Y. Zhang, and J. Luo, Nat. Commun., 9, 2764 (2018).

9. S.F. Yang, N.X. Zhou, H. Zheng, S.P. Ong, and J. Luo, Phys. Rev. Lett., 120, 085702 (2018).

10. J. Luo, Crit. Rev. Solid State Mater. Sci., 32, 67 (2007).

11. J. Luo, J. Materiomics, 1, 22 (2015).

12. T. Frolov, M. Asta, and Y. Mishin, Curr. Opin. Solid St. M., 20, 308 (2016).

13. T. Frolov, M. Asta, and Y. Mishin, Phys. Rev. B, 92, 5 (2015).

14. Y. Mishin, W.J. Boettinger, J.A. Warren, and G.B. McFadden, Acta Mater., 57, 3771 (2009).

15. T. Frolov, S.V. Divinski, M. Asta, and Y. Mishin, Phys. Re. Lett., 110, 255502 (2013).

16. B.-H. Lee, S.-H. Kim, J.-H. Park, H.-W. Kim, and J.-C. Lee, Mater. Sci. Eng. A, 657, 115 (2016).

17. D. Olmsted, L. Hectorjr, and W. Curtin, J. Mech. Phys. Solids, 54, 1763 (2006).

18. W.W. A. Devaraj, R. Vemuri, L. Kovarik, X. Jiang, M. Bowden, J.R. Trelewicz, S. Mathaudhu, A. Rohatgie, Acta Mater., 165, 698 (2018).

19. X. Sauvage, N. Enikeev, R. Valiev, Y. Nasedkina, and M. Murashkin, Acta Mater., 72, 125 (2014).

20. L. Zhang, Y. Shibuta, X. Huang, C. Lu, and M. Liu, Comp. Mater. Sci., 156, 421 (2019).

21. M.I. Mendelev, M. Asta, M.J. Rahman, and J.J. Hoyt, Philos. Mag., 89, 3269 (2009).

22. B. Jelinek, J. Houze, S. Kim, M.F. Horstemeyer, M.I. Baskes, and S.-G. Kim, Phys. Rev. B, 75, 054106 (2007).

23. X.-Y. Liu, P.P. Ohotnicky, J.B. Adams, C.L. Rohrer, and R.W. Hyland, Surf. Sci., 373, 357 (1997).

24. R.I. Babicheva, S.V. Dmitriev, L.C. Bai, Y. Zhang, S.W. Kok, G.Z. Kang, and K. Zhou, Comp. Mater. Sci., 117, 445 (2016).

25. B. Sadigh, P. Erhart, A. Stukowski, A. Caro, E. Martinez, and L. Zepeda-Ruiz, Phys. Rev. B, 85, 184203 (2012).

26. S. Jonathan, S. Alexander, and A. Karsten, Acta Mater., 59, 29572968 (2011).

27. S. Plimpton, J. Comput. Phys., 117, 1 (1995).

28. A. Stukowski, Model. Simul. Mater. Sc., 18, 015012 (2010).

29. M.J. Rahman, H.S. Zurob, and J.J. Hoyt, Metall. Mater. Trans. A, 47a, 1889 (2016).

30. S. Hocker, M. Hummel, P. Binkele, H. Lipp, and S. Schmauder, Comput. Mater. Sci., 116, 32 (2016). 